\title{
Análisis socio-técnico de los regímenes de promoción industrial de 1944 y 1958 en el sector automotriz en Argentina
}

\section{Socio-technical analysis of the industrial promotion regimes of 1944 and 1958 in the automotive sector in Argentina}

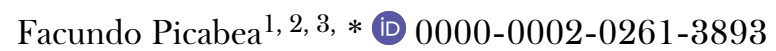

Florencia E. Urcelay ${ }^{2}$ (D) 0000-0002-0261-3893

\footnotetext{
${ }^{1}$ Consejo Nacional de Investigaciones Científicas y Técnicas, Buenos Aires, Argentina.

${ }^{2}$ Universidad Nacional de Luján, Departamento de Ciencias Sociales, Luján, Argentina.

${ }^{3}$ Universidad Nacional de Quilmes, Instituto de Estudios sobre la Ciencia y la Tecnología, Quilmes, Argentina.

*Correspondencia: fpicabea@conicet.gov.ar
}

Resumen. Iniciada la década de 1940, el Estado se constituyó como un actor fundamental en el proceso de industrialización de Argentina. Su participación como interventor, relacionado con la planificación y el control de la economía, se advierte en la promulgación de políticas específicas de promoción industrial. Este artículo analiza los regímenes de 1944 y 1958, plasmados en el Decreto 14.630/44, ratificado como Ley de Industrias de Interés Nacional número 13.892, y la Ley 14.781 Régimen para el Desarrollo Industrial, respectivamente. Asimismo, se analiza la forma en que cada regulación configuró un modelo de cambio tecno-productivo y por lo tanto un tipo de industria; también, analiza comparativamente los modelos de intervención estatal en una rama central del modelo de acumulación de industrialización por sustitución de importaciones y los fundamentos ideológicos que los orientaron.

Palabras clave: Estado intervencionista; políticas públicas; promoción industrial; sector automotor.

Abstract. In the early of 40s, the Argentinian State was constituted like a fundamental actor in the process of industrialization of the country. His participation as an interventor, related to the planning and control of the economy, is notorious for the promulgation of specific industrial promotion policies. This article

CÓMO CITAR: Picabea, F. y Urcelay, F. E. (2019). Análisis socio-técnico de los regímenes de promoción industrial de 1944 y 1958 en el sector automotriz en Argentina. America Latina en la Historia Económica, 26(2), e977. DOI: 10.18232/alhe.977 
analyzes the industrial promotion regimes of 1944 and 1958, regulated by decree $14.630 / 44$, ratified as Industries of National Interest Act number 13.892, and 14.781 Act, Regime for Industrial Development, respectively. Likewise, the way in which each regulation configured a techno-productive change model and therefore a type of industry is analyzed. The paper comparatively analyzes (in each process) the models of state intervention in a central sector of the accumulation model of industrialization by import substitution, as well the ideological foundations that guided them.

Key words: Interventional State; public politics; industrial promotion; automotive sector.

JEL: L62; N16; N46; N66.

Recibido: 24 de marzo de 2018.

Aceptado: 21 de junio de 2018.

Publicado: 30 de enero de 2019.

Organismo patrocinador: Consejo Nacional de Investigaciones Científicas y Tecnológicas, Argentina.

\section{INTRODUCGIÓN}

A mediados del siglo xx, el Estado se constituyó como un actor fundamental en el proceso de industrialización de Argentina. Su intervención directa es manifiesta a partir de una serie de instrumentos como las políticas de promoción industrial y la selección de áreas consideradas estratégicas para ser fomentadas. Entre esos mecanismos destacan las leyes que orientaron regímenes específicos de promoción, por los cuales podemos dar cuenta de las variaciones en el modo de regulación.

El objetivo del presente artículo es analizar comparativamente los regímenes de promoción industrial de 1944 (Decreto 14.630/44, ratificado como Ley de Industrias de Interés Nacional número 13.892) y 1958 (Ley 14.781 Régimen para el Desarrollo Industrial), con especial interés en la generación de capacidades locales. El análisis de ambos instrumentos tiene cuatro objetivos: a) analizar las características principales de cada régimen de promoción y las normas específicas dirigidas a la industria automotriz; $b$ ) analizar las transformaciones del sector automotriz a partir de los cambios en el modo de regulación; $c$ ) evaluar alcances y límites de cada régimen a partir de la generación de capacidades a nivel sectorial, y $d$ ) realizar un balance sobre los modelos de industrialización en países en desarrollo.

Para comprender mejor el alcance de cada régimen, se analizará un caso específico: la industria automotriz. Este sector constituye un estudio de caso representativo del marco regulatorio debido a que la fabricación de automóviles fue un elemento clave en el desarrollo de la industria argentina, excediendo incluso el recorte temporal aquí analizado 1

\footnotetext{
${ }^{1}$ La industria automotriz constituye un sector con una alta capacidad para generar eslabonamientos económicos y movilizar tanto la extracción de materias primas como la producción de bienes intermedios. Por ello ha mostrado acabados resultados positivos en el proceso de industrialización de países en desarrollo durante el siglo xx. Esto ocurrió tanto en Europa (en los países menos desarrollados, pero también en Francia y Alemania luego de la segunda guerra mundial), como en Asia o en América Latina, donde el sector automotriz fue central para promover el desarrollo de toda la industria nacional. Para un estudio específico de industria automotriz en países en desarrollo, véanse Baronson (1971), Biggart y Guillen (1999), Carvalho, Ferraz y Kupfer (2009), Catalan (2010). Para un análisis del sector en Argentina, véanse Sourrouille (1980), Belini (2006, 2009), Harari (2007, 2015), Ianni (2008), Picabea (2012a, 2012b), Raccanello (2013), Picabea y Thomas (2015a, 2015b).
} 
El marco temporal de este trabajo se extiende desde 1943 hasta 1966. El periodo inició con el golpe de Estado dirigido por el Grupo de Oficiales Unidos (GOU) y concluyó con el golpe de Estado que derrocó al presidente Arturo Illia, la autodenominada revolución argentina. El análisis abarca etapas clave de la historia del siglo xx argentino, permite comparar dos regímenes de promoción sectorial y cuestionar la periodización tradicional que marca una división en el proceso de industrialización por sustitución de importaciones entre 1955 y 1958, minimizando las implicancias de la creación de una fábrica estatal de automotores en el segundo gobierno peronista. Como se observará más adelante, sostener que la industria automotriz argentina inició en 1952 permite poner en discusión dicha distinción.

El proceso de industrialización en Argentina ha sido estudiado por numerosos autores desde muy diversos enfoques, privilegiando el análisis de variables macro de la estructura económica, del modelo de industrialización implementado, de sectores industriales específicos o del desarrollo de casos particulares ${ }^{2}$ En general, estos trabajos presentan restricciones en dos niveles: $a$ ) la reducción de la complejidad de los procesos de cambio social y tecnológico y b) la relativización de los resultados de la instauración de la política industrial del gobierno peronista respecto de la estructura tecno-productiva y su significación para la posterior radicación de empresas transnacionales, durante el desarrollismo.

Si bien existe una amplia y diversa biblioteca sobre la economía y la industrialización durante el peronismo y el desarrollismo, puede afirmarse que no se han investigado en profundidad las consecuencias en términos de generación de capacidades locales del proceso de promoción e implementación de la política industrial orientado a transformar la estructura tecno-productiva local. Ello permitiría comprender mejor no sólo las estrategias explícitas (las políticas públicas), sino también sus resultados en el modelo de acumulación y cambio tecnológico desarrollado entre 1944 y 1966.

Por ello este artículo apunta a identificar nuevas relaciones, reconstruir nuevos procesos y generar nuevas explicaciones, a partir de la triangulación de conceptos de economía política y del enfoque constructivista de la sociología de la tecnología, integrados en un marco específico cuyos elementos se exponen a continuación.

El enfoque de la economía política considera al Estado no como un espacio político neutral, ni el mero representante de una monolítica clase dominante, sino que en él se dirimen los distintos intereses de las fracciones que la componen; por ello se movilizan y cristalizan alianzas inter e intraclase y expresa, en cada configuración, las relaciones de fuerza de las clases sociales (Picabea y Thomas, 2015a).

\footnotetext{
${ }^{2}$ Sobre la política económica e industrial argentina pueden consultarse numerosos trabajos ya tradicionales. Los más relevantes, en general, son estudios de largo plazo que han contribuido a sentar los marcos interpretativos del proceso y crear agendas de investigación. Entre ellos, los más destacados cronológicamente son Ferrer (2004); Altimir, Santamaría y Sourrouille (1967); Díaz Alejandro (1970); Jorge (1971); Di Tella y Zymelman (1973); Di Tella (1973); Mallon y Sourrouille (1973); Llach (1984); Schvarzer (1996); Gerchunoff y Llach (2003) y Basualdo (2006). Aun cuando estos trabajos responden a enfoques diversos e incluso opuestos (neoclásicos, estructuralistas y marxistas), todos coinciden en señalar la importancia del peronismo para consolidar la primera fase del proceso de industrialización por sustitución de importaciones y dedican muy poco espacio a analizar los procesos de desarrollo tecno-productivo a partir de 1950 , considerando, de esta forma que es a partir de 1955 o 1958 (dependiendo de los autores), el momento en que inicia la segunda fase de industrialización. Más recientemente, desde la perspectiva de la historia industrial y haciendo hincapié en estudios de caso sectoriales, se destacan algunos trabajos pioneros sobre el desempeño empresarial del Estado en Argentina de posguerra, que recuperan aspectos originales de la relación entre industria y Estado durante el primer peronismo y le otorgan mayor relevancia al desarrollo de una industria más compleja a partir de 1950 a partir de la planificación del Estado y la coordinación del proceso (Belini y Rougier, 2008; Belini, 2006, 2009).
} 
Por modelo de acumulación se entiende en primer lugar el conjunto de características económicas, políticas y sociales de un determinado periodo histórico, que se articulan para favorecer la acumulación y reproducción del capital. Por otra parte, de acuerdo con la definición de Arceo (2003), "las características del modo de acumulación dependen de la estructura económico-social, de las luchas políticas y sociales que fueron conformando esa estructura y de la composición del bloque de clases que deviene dominante y que impone un sendero de acumulación acorde a sus intereses" (p. 19).

Una dinámica socio-técnica es un conjunto de patrones que organiza la interacción entre tecnologías, instituciones, racionalidades e ideología. Una dinámica socio-técnica incluye un conjunto de relaciones tecno-económicas y socio-políticas vinculada a un proceso de cambio tecnológico (Thomas, 2006). Una trayectoria socio-técnica es un proceso de coconstrucción de productos, procesos productivos y organizacionales, instituciones, relaciones usuario-productor, procesos de aprendizaje, relaciones problema-solución, procesos de construcción de funcionamiento/no funcionamiento de una tecnología, racionalidades, políticas y estrategias determinadas (Bijker, 1995).

El estilo socio-técnico puede conformarse por distintos factores, entre ellos, la construcción de prestigio para un gobierno, factores de naturaleza geográfica, o experiencias históricas regionales y nacionales (Thomas, 2008). La resignificación de tecnologías, como un estilo socio-técnico, constituye operaciones de reasignación de sentido de una tecnología y de su medio de aplicación. Resignificar tecnologías implica refuncionalizar los conocimientos, artefactos y sistemas, así como la utilización creativa de las tecnologías ya disponibles (Thomas, 1999). El concepto permite explicar instancias fundamentales en la producción de tecnologías conocimiento-intensivas en el ámbito local.

Los conceptos de desarrollo de impulso endógeno y exógeno tienen por objeto establecer una distinción entre dos estilos socio-técnicos asociados a los regímenes de promoción industrial (Picabea, 2012a). Por impulso endógeno se entiende la identificación, resignificación y generación de condiciones tecno-productivas (capacidades, recursos humanos, infraestructura, capitales, etc.), a partir de recursos locales como empresas públicas y privadas. Por el contrario, por impulso exógeno se entiende la estrategia de incorporar a la estructura tecno-productiva local, capacidades ya generadas por empresas extranjeras en sus países de origen.

\section{RÉGIMEN DE INDUSTRIAS DE INTERÉS NACIONAL: PRIMER SISTEMA DE FOMENTO INDUSTRIAL DEL PAÍS}

Las políticas públicas tendentes a promover el cambio tecno-productivo y un correspondiente desarrollo económico en Argentina tuvieron tres canales principales a través de los cuales se llevaron a cabo. En primer lugar, la promoción industrial estricta, leyes especiales que regulaban preferencias estatales y privilegios a empresas, sectores y/o regiones; luego la creación del Banco Industrial, una entidad bancaria dedicada exclusivamente a proveer de capitales al sector y, por último, la transferencia de recursos del Estado (insumos e infraestructura) al sector industrial privado.

El decreto del poder ejecutivo nacional número 14.630, sancionado en 1944, significó el primer sistema de fomento de la industria nacional de Argentina. Posteriormente convertido en ley (13.892), el objetivo de este decreto fue establecer un régimen de fomento y defensa para un conjunto de industrias que pasó a denominarse de interés nacional, definidas como aquellas que utilizaban materias primas nacionales y su producción estaba dirigida al mercado interno, o que utilizando de manera parcial o total materias primas importadas, producían artículos de primera 
necesidad, y las asociadas a la defensa del país. De esta manera quedaban establecidos parámetros amplios para la selección de industrias a fomentar y no se definieron privilegios hacia ninguna rama específica, teniendo en cuenta el predominio de la industria liviana hasta ese momento 3

Las industrias incluidas en el régimen fueron beneficiadas con tres tipos de incentivos que actuaron como protección contra los productos importados y fomento para sectores aún no desarrollados: derechos aduaneros adicionales de fomento y de defensa (estos últimos planificados para productos afectados por maniobras de dumping), cuotas de importación y subsidios para casos previamente analizados.

Además, se dispuso la creación de una comisión asesora de fomento industrial, encargada de evaluar y medir la aplicación de los instrumentos en cada industria propuesta, tanto por empresarios como por empresas industriales públicas. La Comisión era quién elevaba sus informes (por medio de la Dirección General de la Industria), al Ministerio de Agricultura, para la aprobación de cada caso estudiado por parte del ejecutivo nacional. La Secretaría de Industria realizaba en paralelo su propio estudio del sector propuesto y enviaba los resultados al poder ejecutivo, quien disponía la aceptación o rechazo del expediente (Belini, 2010).

Entre las bases del decreto se destaca la definición dela función del Estado como garante del desarrollo económico y la consideración de la industria como una actividad de vital importancia para el país, que no obstaculizaba la producción agropecuaria, ya que proponía incentivar la elaboración de materias primas naturales:

Es deber primordial del Estado fomentar y asegurar el desarrollo de su economía, favoreciendo la evolución y perfeccionamiento de los múltiples factores que la integran, [...] la importancia que la industria nacional ha adquirido se revela por el hecho de que el valor que agrega a las materias primas iguala al conjunto de la producción neta agrícola ganadera [...] [y] el incremento del desarrollo industrial no obstaculiza a la producción agropecuaria y es compatible.... (Decreto 14.630/44) $4_{4}^{4}$

En general, la bibliografía caracteriza al régimen de promoción industrial del peronismo como un marco tímido que "combinó una serie de instrumentos a fin de alentar el crecimiento manufacturero [pero que] no conformó una política industrial” (Belini, 2009, p. 11). A su vez, se afirma que el propio decreto citado no constituía una herramienta suficiente puesto que reconocía al sector agrario como base de la economía de Argentina. Sin embargo, una lectura atenta del fragmento citado, y menos ortodoxa del régimen -que considere el propósito estratégico del desarrollo local, por ejemplo-, permite confirmar que se trata del primer instrumento específico de fomento industrial en la historia del país.

La idea de resaltar el carácter no contradictorio entre industria y agricultura puede considerarse como una estrategia, tanto para conformar a los grupos rurales, como para reafirmar la necesidad de mantener, e incluso impulsar, la exportación de bienes primarios para la financiar el proceso. El régimen de 1944, aun cuando se presentó como un marco regulatorio complementario de la economía agropecuaria su potencial era muy alta y fue, por sus propios resultados, un hito para el cambio en el modelo de acumulación y desarrollo tecno-productivo. Y se mantuvo como el único régimen de promoción industrial nacional hasta 1958.

\footnotetext{
${ }^{3}$ Para un análisis del decreto de industrias de interés nacional desde su elaboración, antecedentes y actores involucrados, véase Belini (2010, 2014).

${ }^{4}$ Recuperado de http://cdi.mecon.gob.ar
} 
GUADRO 1. INDUSTRIAS DECLARADAS DE INTERÉS NACIONAL

\begin{tabular}{lrllc}
\hline Año & Decreto & Industria & $\begin{array}{c}\text { Criterios } \\
\text { de clasificación }\end{array}$ & $\begin{array}{c}\text { Vigencia } \\
\text { de beneficios (años) }\end{array}$ \\
\hline 1945 & 29.246 & Azufre & Defensa & 5 \\
1946 & 6.67 & Arrabio & Defensa & 2 \\
1946 & 7.219 & Maderas terciadas & Defensa & s. d. \\
1946 & 12.006 & Pilas eléctricas húmedas & Establecida & 2 \\
1946 & 5.447 & Ácido cítrico & Fomento & 2 \\
1946 & 19.339 & Cloruro de bario & Establecida & 2 \\
1946 & 21.743 & Carreteles de madera & Establecida & 1 \\
1947 & 40.948 & Carburo de tungsteno & Defensa & s. d. \\
1947 & 5.687 & Hierro laminado & Establecida & 2 \\
1947 & 7.295 & Agua oxigenada & Establecida & 1 \\
1947 & 10.993 & Penicilina & Fomento & $5^{\mathrm{a}}$ \\
1947 & 34.285 & Película virgen & Fomento & 1 \\
1947 & 37.452 & Fibras vegetales largas & Fomento & 5 \\
1947 & 7.406 & Fieltros y asfaltinas & Establecida & 2 \\
1948 & 574 & Materiales fotográficos sensibles & Establecida & 5 \\
1948 & 36.114 & Sulfamidas & Fomento & 5 \\
1948 & 36.108 & Sulfato de bario (baritina) & Establecida & 3 \\
1948 & 39.507 & DDT y composiciones insecticidas & Fomento & 5 \\
1949 & 25.788 & Productos plásticos sintéticos & Establecida & 3 \\
1949 & 27.281 & Tintas gráficas & Establecida & 3 \\
1949 & 31.436 & Herramientas en general & Establecida & 3 \\
1949 & 2.68 & Película radiográfica & Establecida & 5 \\
1950 & 13.848 & Pólvora, explosivos y afines & Defensa & 3 \\
1950 & 19.265 & Dolomita calcinada & Establecida & 2 \\
\hline
\end{tabular}

a Prorrogado por cinco.

Fuentes: elaboración propia con base en Altimir, Santamaría y Sourrouille (1967) y Belini (2010).

En otro nivel, las leyes de promoción alentaron una mayor diversificación tecno-productiva al promover la instalación de nuevas industrias químicas, siderometalúrgicas y eléctricas (Belini, 2010, p. 61). A lo largo del periodo en que estuvo vigente este régimen, entre 1944 y 1957, se declararon de interés nacional 39 industrias (véase cuadro 1 ).

El aumento de la complejidad tecno-productiva de las industrias declaradas de interés nacional fue evidente; mientras que en los primeros años de la década de 1940 se estima principalmente la elaboración de ciertas materias primas (azufre, arrabio, maderas terciadas, ácido, etc.), en 1947 comienzan a declararse industrias de bienes intermedios (productos plásticos sintéticos, tintas gráficas, herramientas en general, película radiográfica, pólvora, explosivos y afines, etc.), y a comienzos de la década de 1950 se declaran de interés nacional ramas completas de la industria (automotores, caños sin costura, etc.). Ello muestra una dinámica socio-técnica en proceso de transformación, volviéndose más basta, densa y con vínculos a nivel local. 
En el marco del régimen de promoción de 1944, en 1951, a través del decreto 25056/51 el Estado declaró a la industria de automotores y de maquinaria e implementos agrícolas y sus repuestos y accesorios de interés nacional. El decreto consideró a la industria automotriz como una industria a fomentar, de vital importancia para la economía y la defensa del país, y justificó su incorporación al régimen establecido en 1944 debido a la significación adquirida: "en cuanto al número de establecimientos, capitales invertidos, personal ocupado, capacidad de producción y técnica lograda" (Decreto Ley 25.056/51)

El propósito de la integración de la industria automotriz fue que junto con la de maquinarias e implementos agrícolas, accediera a los privilegios que establecía el régimen de industrias de interés nacional. En especial a los beneficios de permisos previos y cuotas de importación para materias primas, equipos e instrumentos que no se fabricaran en el país o no estuvieran disponibles de manera adecuada, por cantidad, calidad o precio. El poder ejecutivo tenía una función determinante en la aplicación del régimen: el Ministerio de Industria y Comercio y el Ministerio de Hacienda establecerían las formas y condiciones para acceder a permisos previos o cuotas de importación; el Ministerio de Hacienda vigilaría el destino final de las materias primas, equipos e instrumentos importados; el Ministerio de Finanzas otorgaría los permisos de importación y el Ministerio de Industria y Comercio crearía un registro en el cual debían inscribirse los establecimientos que se acogieran a los beneficios, además de ser el encargado de proponer la renovación o modificación de los mismos luego del plazo establecido de cinco años. Finalmente, el régimen fue prorrogado por tres años más en 1957, mediante el Decreto 11.954/57 (Altimir, Santamaría y Sourrouille, 1967).

Lo que quedó establecido mediante estas regulaciones fue un sistema de promoción que pretendía alcanzar los objetivos de desarrollo industrial mediante el impulso endógeno de infraestructura y capacidades tecno-productivas. Es decir, la generación local de conocimientos, procesos y artefactos, con el fin de aproximarse al marco tecnológico de los países centrales y alcanzar a su vez una mayor autonomía respecto al mainstream internacional.

\section{La creación del sector automotriz en el proceso de industrialización}

En la década de 1950, en Argentina, al igual que en el resto de los países latinoamericanos, el parque automotor local se había generado a partir de la importación de vehículos fabricados en el exterior. Si bien se importaban vehículos desde principios del siglo xx, la dinámica socio-técnica de producción mundial de las empresas transnacionales desarrolló un estilo basado en la fabricación de partes en casas matrices y el armado en los países de destino de los artefactos (Belini, 2006). Esto se inauguró en Argentina con la instalación de la empresa Ford en 1913, a la cual le siguió General Motors en 1925 y Chrysler en 1932. Este sistema, denominado ensamblado, no se vinculó con la estructura tecno-productiva local ya que, al importar los vehículos completos, pero desarmados, tenía una participación casi nula de componentes locales en la matriz insumo-producto (Picabea y Thomas, 2015a).

La experiencia más importante de radicación de empresas extranjeras a partir del régimen de promoción industrial en este periodo fue una pequeña empresa italiana llamada Cisitalia, rebautizada como Automotores Argentinos S. A. (Autoar). La empresa intentó la fabricación de varios

\footnotetext{
${ }^{5}$ Anales de Legislación Argentina XI, Colegio Público de Abogados.
} 
modelos sobre la base de las matrices importadas y con un grado de integración elevado. No obstante, su producción de un centenar de unidades declinó a finales del peronismo (Belini, 2006, p. 116).

A comienzos de la década de 1950, el parque automotor argentino evidenciaba un importante retroceso respecto a las primeras décadas del siglo, con una clara tendencia a la baja de la importación durante en apenas 30 años. En general, este aspecto es analizado por la bibliografía mainstream como una consecuencia de la política económica proteccionista que no dio lugar a la demanda. Sin embargo, la caída en las importaciones está mucho más asociada a cuestiones externas de oferta, como las restricciones productivas y comerciales durante la crisis de $1930 \mathrm{y}$ la reorientación de la industria estadunidense y europea durante la segunda guerra mundial y la posguerra (véase gráfica 1).

En el escenario de posguerra, con muy poca oferta de automóviles por parte de las empresas transnacionales y los problemas de balanza de pagos, la producción local de automotores se volvió una estrategia central para el gobierno peronista. El automóvil de producción local se presentaba como una solución que apuntaba tanto a resolver la demanda de automotores como el desarrollo económico, generando una dinámica socio-técnica compleja e integrada. Los funcionarios pensaban que la producción local favorecería la integración de recursos materiales, empresariales y la fuerza laboral, contribuiría con la estructura tecno-productiva, promovería el desarrollo industrial auto-sostenido y servía como materialización de los proyectos del gobierno.

En 1952, a través del Decreto 6191/52, se creó Industrias Aeronáuticas y Mecánicas del Estado (en adelante IAME), empresa sometida al régimen de la Ley 13.653 de Empresas del Estado y dependiente del Ministerio de Aeronáutica. IAME se financió en su primera fase con un crédito del Banco Industrial de la República Argentina de 53000000 pesos (unos 3000000 dólares, indexados de acuerdo al Banco Central de la República Argentina). En relación con el régimen vigente, IAME se vio beneficiada por el establecimiento de cuotas de importación y la liberación de derechos para la introducción de maquinaria e insumos. Fue el propio Estado quien generó las condiciones para este proyecto y lideró su ejecución.

IAME se constituyó dentro de las dependencias del Instituto Aerotécnico, ex Fábrica Militar de Aviones, la institución pionera en la industria aeronáutica nacional que había desarrollado hacia 1950 un estilo de diseño y producción impulsando la creación de numerosos proveedores locales (Lalouf, 2005). En el artículo tercero del decreto de creación se estableció que iame reemplazaría al Instituto Aerotécnico, integrando su personal, gabinetes, talleres, fábricas y todas las instalaciones y dependencias Dirección Nacional de Fabricaciones e Investigaciones Aeronáuticas [DINFIA], 1967). Los artefactos producidos fueron resultado de una estrategia de desarrollo industrial por sustitución de importaciones que apuntaba a generar un nuevo sector productivo central en el modelo de acumulación, mientras se buscaba cubrir diferentes segmentos del mercado automotriz, atendiendo a las demandas de artefactos de diferentes grupos de usuarios. Para el diseño y producción de automóviles se implementó un estilo socio-técnico de resignificación de tecnologías. Es decir, el Estado usó los recursos con los que inicialmente contaba y les asignó nuevos sentidos y utilidades: la fábrica de automotores de IAME se montó sobre la estructura de la antigua fábrica militar de aviones (tanto la infraestructura como conocimientos y recursos humanos); los vehículos se diseñaron a partir de artefactos modélicos (Moto Puma, Justicialista y tractor Pampa) o bien fueron consecuencia de la reutilización de partes de otros (Rastrojero). Uno de los aspectos más 
GRÁFICA 1. DINÁMICA DE LA IMPORTACIÓN

DE VEHÍCULOS EN ARGENTINA (1920-1949)

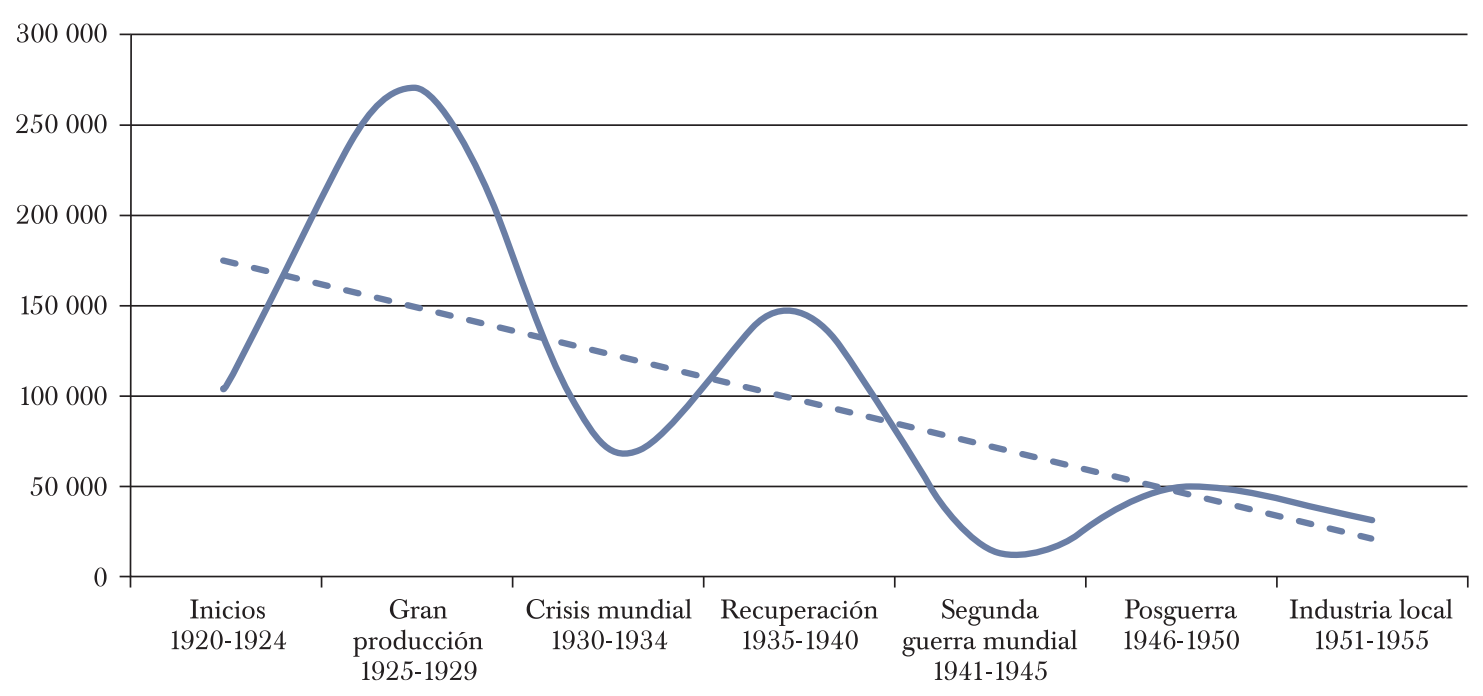

Fuentes: elaboración propia con base en Adefa (1966).

destacados de la creación de iAME (y anteriormente del Instituto Aerotécnico), fueron sus áreas de I+D puesto que, aun con algunas reservas sobre el estilo, el diseño de nuevos artefactos era clave en una dinámica socio-técnica de desarrollo endógeno de capacidades.

Uno de los proyectos emblema fue la fabricación de un utilitario para el campo, el Rastrojero. El proyecto, que comenzó de manera informal y a partir de inquietudes personales de algunos altos funcionarios del gobierno nacional, se convirtió en poco tiempo en el utilitario más vendido del mercado por casi 30 años ${ }^{6}$ Entre 1953 y 1955 se fabricaron en IAME 18300 vehículos (sumando los diferentes artefactos y sus modelos). La producción de estos bienes promovió la creación de eslabonamientos económicos en una mediana red de proveedores de insumos y partes componentes que desarrollaron una infraestructura industrial.

En 1953, cuando las actividades de IAME comenzaban a mostrar resultados positivos en relación con el proyecto metalmecánico, la idea de recurrir a empresas extranjeras para acelerar el proceso de producción local de automotores volvió a tener vigencia. Los funcionarios del gobierno consideraban que las empresas extranjeras con experiencia en la industria automotriz podían funcionar como aceleradores de la maduración del sector.7 ${ }^{7}$ La estrategia fue la modificación de las regulaciones y convenios privilegiados, pero manteniendo a estas empresas extranjeras muy cerca del Estado.

La sanción de la Ley de Inversiones Extranjeras en agosto de 1953 impuso un régimen que le permitió a un grupo de empresas acogerse a beneficios específicos. Una de esas empresas fue Concord, creada a partir del acuerdo entre Fiat y el gobierno nacional para la fabricación de tractores

${ }^{6}$ En 1951, el presidente Juan Domingo Perón desafió al brigadier Juan Ignacio San Martín, ex director del Instituto Aerotécnico y por entonces ministro de Aeronáutica, a hacer un vehículo con los tractores Empire retirados de circulación. San Martín aceptó el reto y llevó una de las unidades a los talleres del Instituto Aerotécnico (Picabea y Thomas, 2015a).

${ }^{7}$ Entrevista al Ing. Monserrat, José Higinio, director Fábrica de Motores y Automotores IAME: mayo de 2008. 
(Sourrouille, 1980). Dicho acuerdo estableció, además de la transferencia (cuasi llave en mano), de la producción de tractores de IAME, el otorgamiento de divisa por parte del Banco Central para la importación de equipos, materias primas y maquinaria.

Otro ejemplo del accionar directo del Estado durante el peronismo respecto a capitales extranjeros, fue el acuerdo establecido con la empresa estadunidense Kaiser-Willlys Corp. para la instalación en el país de una fábrica de automóviles, camionetas y Jeeps en enero de 1955. La empresa se instaló en Córdoba bajo el nombre de Industrias Kaiser Argentina S. A. (en adelante IKA) y constituyó junto con Fiat-Concord, los ejemplos de empresas mixtas. Además, estos acuerdos confirman la relevancia de IAME en el desarrollo de la infraestructura para la generación de la industria automotriz privada. En ambos casos, la radicación de las firmas extranjeras tan vinculadas a la industria local (estatal y privada), promovía activamente el desarrollo endógeno de capacidades a partir de una dinámica socio-técnica colaborativa más que competitiva.

El crecimiento de la industria automotriz se dio como consecuencia de la regulación de 1944 y la declaración de interés nacional en 1951, con la creación de IAME, primero, y la radicación de Fiat, Mercedes Benz e IKA, después. De esta forma, la producción local de automóviles pasa de menos de una veintena en 1951 a 18290 en 1959. Por su parte, el sector utilitario, liderado por IAME e IKA, pasó de 90 a 13826 en el mismo periodo (véase cuadro 2). Más allá de los resultados cuantitativos (que pueden considerarse insuficientes para dar cuenta de los requerimientos del mercado), el diseño y la fabricación de automóviles (que implicó a su vez el diseño del layuot tecno-productivo), favoreció significativamente la generación de capacidades fundamentales para la industria nacional.

El régimen de promoción establecido entre 1945 y 1957 permite afirmar que este primer marco regulatorio (compensado con otros elementos de política pública sectorial), alentó el proceso de industrialización. A partir de un estilo socio-técnico de impulso endógeno, basado en la resignificación de tecnologías, el Estado aprovechó buena parte de la infraestructura y experiencia en otras ramas para alentar la generación local de capacidades: se reutilizaron empresas públicas; se dinamizó el Banco Industrial para el financiamiento del sector privado; se promovió la creación de recursos humanos sectoriales a partir del impulso de la educación técnica; se asesoró, capacitó e incluso se equipó al sector privado; se declararon de interés nacional industrias proveedoras del sector; se creó una empresa pública que diseñó y produjo automotores 8

\section{EL RÉGIMEN DE FOMENTO INDUSTRIAL DE 1958: INVERSIÓN EXTRANJERA DIRECTA Y RADICACIÓN DE EMPRESAS TRANSNACIONALES}

El sistema de fomento industrial establecido por el gobierno de Arturo Frondizi en diciembre de 1958 no puede ser entendido únicamente a partir de la Ley de desarrollo de la industria número 14.781, sino que debe analizarse junto a la Ley 14.780, que estableció el régimen legal para las inversiones de capitales extranjeros. Como indican Katz y Kosacoff (1989), ambas leyes y un

\footnotetext{
${ }^{8}$ Luego del golpe de estado de 1955, a través del Decreto-Ley 142/55, el gobierno de facto restituyó a la planta industrial de IAME el antiguo nombre de Fábrica Militar de Aviones, lo que puso de manifiesto la idea de quitarle al Estado su protagonismo en la industria automotriz. Esta política se hizo evidente cuando los interventores comenzaron paulatinamente a cancelar todos los proyectos de I+D no vinculados directamente a aeronáutica o a vehículos comercializables en el mercado. Todos los desarrollos experimentales y ensayos técnicos fueron suspendidos primero y cancelados después.
} 


\section{CUADRO 2. PRODUCGIÓN AUTOMOTRIZ ARGENTINA AL AMPARO DE LA REGULACIÓN DE 1944}

\begin{tabular}{lcccccccccc}
\hline Producción & 1951 & 1952 & 1953 & 1954 & 1955 & 1956 & 1957 & 1958 & 1959 & Totales \\
\hline Automóviles & 18 & 62 & 63 & 173 & 235 & 326 & 5461 & 14310 & 18290 & 38938 \\
Utilitarios & 90 & 69 & 2011 & 2621 & 4530 & 5039 & 10167 & 13523 & 13825 & 51875 \\
$\begin{array}{c}\text { Camiones } \\
\quad \text { y colectivos }\end{array}$ & 0 & 838 & 1000 & 565 & 1626 & 578 & 7 & 1 & 837 & 5452 \\
\hline
\end{tabular}

Fuentes: AdEFA (1966) y Sourrouille (1980).

conjunto de reglamentaciones sectoriales conformaron el marco legal en el que se desenvolvió la promoción industrial, en lo que tradicionalmente se considera una nueva etapa de profundización del modelo sustitutivo de importaciones.

Si bien el modelo de acumulación continuó siendo sustitutivo y mercado-internista, el nuevo marco regulatorio para la promoción industrial implicó un nuevo modo de regulación. A partir de 1958, un conjunto de variables muestra que el desarrollo de la estructura tecno-productiva se orientó sobre la base del impulso exógeno, es decir, el desarrollo de capacidades a partir de la radicación de empresas transnacionales, que trasladaban a la estructura local las capacidades generadas en sus países de origen. La ley de inversión de capitales extranjeros fue el instrumento que coordinó el proceso. Promulgada el 22 de diciembre de 1958, la Ley 14780 estableció que los capitales extranjeros que fueran invertidos en actividades productivas nuevas o existentes tendrían los mismos derechos que los de origen nacional: "Los capitales extranjeros que se inviertan en el país en la promoción de nuevas actividades productivas y en la ampliación y/o perfeccionamiento de las existentes, necesarias para el desarrollo económico nacional gozarán de los mismos derechos que la Constitución y las leyes acuerdan a los nacionales" (Ley 14780, INFOLEG-MEN).

Los capitales podían ingresar al país en forma de divisas o máquinas, equipos o instalaciones, previa autorización del poder ejecutivo y debían sujetarse a "proporcionar suficientes garantías técnico económicas y contribuir directa o indirectamente, a sustituir importaciones, incrementar las exportaciones o promover aquellos desarrollos conducentes a un racional y armónico crecimiento de la economía nacional" (Ley 14780, INFOLEG-MEN)

Esta ley también establecía que se otorgarían preferencias a los capitales dirigidos al desarrollo e integración de las economías regionales, la elaboración de materias primas, la producción de bienes de capital y de utilización intermedia y aquellos que se asociaran a empresas de capitales nacionales. Además, habilitaba al poder ejecutivo a acordar sobre diversos beneficios como derechos aduaneros, régimen impositivo cambiario, tratamiento crediticio e inclusión en regímenes específicos de fomento de la industria. respecto al impacto que significaba esta apertura de capitales a las empresas nacionales ya establecidas, la ley sólo indica que el poder ejecutivo debía observar que no afectaran el desenvolvimiento de las mismas, y a su vez, que tuvieran asegurada la posibilidad de importar equipos o elementos de producción.

Por su parte, la Ley 14.781 estableció específicamente el régimen de fomento industrial y tuvo como objetivo promover un "desarrollo integral y armónico" de la producción del país. Los propósitos concretos que conllevaba esta meta se dirigían a perfeccionar, ampliar y diversificar la producción industrial, descentralizándola y aprovechando los recursos actuales y potenciales del 
país e incentivando la promoción tecnológica en la producción, con miras de lograr un equilibrio en la balanza de pagos del exterior. Además, se menciona la defensa nacional, la salud y la seguridad pública como aspectos esenciales.

Para alcanzar los objetivos propuestos, la ley dispuso la liberación de derechos y adicionales aduaneros a la importación de maquinarias y equipos; imposición de derechos aduaneros o alza en caso de que existieran a los productos importados que afectaran el desarrollo de la producción local; suspensión o limitación de importaciones de productos elaborados y materias primas que se produzcan en el país; tratamiento cambiario preferencial para exportaciones industriales; otorgamientos de créditos y medios de financiación para planes industriales; suministro preferencial de materias primas, energía, combustibles y transporte; tratamiento preferencial en las compras por organismos del Estado; y exención o desgravación de impuestos por periodos limitados (Ley $14781 / 58)$.

Como política complementaria, el gobierno de Artuto Frondizi creó el Consejo Nacional de Promoción Industrial, como asesor de la Secretaría de Estado de Industria y Minería, con el fin de elaborar dictámenes, estudios y proyectos para el fomento de determinadas industrias, es decir, fue el organismo encargado de instrumentar las leyes. Ambas regulaciones se caracterizan por la amplitud de los objetivos a cumplir y la falta de precisión en el establecimiento de beneficios, destinatarios y controles. En este marco, la definición concreta del régimen se realizó a través de reglamentaciones que establecieron un criterio selectivo de actividades que serían prioritarias. Como indican Katz y Kosacoff (1989, p. 42), estas reglamentaciones abarcaron sectores como la industria siderúrgica (Decreto 5.038/61), a la industria petroquímica (Decreto 5.039/61) y a la industria celulósica (Decreto 5.041/61), además de planes regionales para la Patagonia, la región Noroeste y la provincia de Corrientes.

El poder ejecutivo quedaba ampliamente facultado para diseñar la política de promoción dentro de estos amplios límites impuestos por las leyes (Altimir, Santamaría y Sourrouille, 1967). Por su parte, Schvarzer (1987, p. 13) señala que "la indefinición de las exigencias a los inversores se combinaba con la amplitud conferida para el otorgamiento de los beneficios; no había restricciones claras en ninguno de ambos casos", e incluso la ley de inversión de capitales extranjeros no establecía ningún control o criterio de evaluación para la importación de bienes naturales, ni restringía el acceso a bienes industriales usados u obsoletos por su tecnología. A su vez, la promoción de importación de insumos intermedios y bienes de capital específicos, desestimulaba su producción a nivel local y si bien la radicación generaba algunas dinámicas socio-técnicas con proveedores locales, el marco regulatorio promovía la instalación de proveedores internacionales por lo que disminuía cuantitativa y cualitativamente los procesos de aprendizaje. Finalmente, la radicación masiva de empresas extranjeras (véase cuadro 3), generó una sobre oferta de bienes que condicionó la creación de empresas de capital local, que pocos años después de su creación dejaron de operar.

De esta forma, al propio diseño del instrumento, que dejaba muchos aspectos librados a la consideración de los actores (en general empresas transnacionales), se sumaba el carácter laxo de la regulación. Las fábricas de automotores instaladas o a instalarse debieron seguir las normas establecidas por el Decreto 3.693, de 1959. Conocido como el Régimen de Promoción de la Industria Automotriz, fue aplicado por la Secretaría de Industria y Minería que definió como industria automotriz a "todo establecimiento que integre en su planta una unidad automotriz completa en la que intervengan partes de industria nacional y partes importadas" (Decreto 3.693/59, INFOLEG-MEN). Para ser incluidas en el régimen las empresas debían cumplir una serie de condiciones enumeradas en el decreto: acreditar capacidad técnica y financiera; presentar un plan de producción no menor 
a cinco años; los equipos y/o maquinarias que se importaran debían no ser producidos en el país en calidad y entrega aceptable; y ser totalmente nuevos, salvo en caso de equipos especiales y de difícil disponibilidad, que se destaquen por su alto rendimiento y eficiencia; la producción debía incorporar los adelantos tecnológico contemporáneos. La falta de capacidad o interés del Estado en el control de calidad de los equipos e instalaciones importados implicó que las empresas transnacionales finalmente instalaran en el país maquinaria con décadas de uso en sus países de origen o sobrevaluadas, puesto que se trataba de bienes ya amortizados.

Esto fue más explícito en la industria automotriz. En 1958, las autoridades de IKA reclamaron al gobierno desarrollista que el nuevo régimen de promoción era poco exigente y no establecía incentivos a las terminales para articularse con la industria auxiliar: "La laxitud que ofrece el régimen tal vez explique el interés de tantas firmas por acogerse a sus disposiciones, que configuran una excepción notable al régimen de recargos, en tanto la liberalidad con que han sido autorizadas todas esas firmas va contra la experiencia mundial en la industria, que tiende a concentrarse en establecimientos de gran envergadura" (IKA, 1960).

Para estas empresas el beneficio consistía en la eliminación del recargo de $300 \%$ que pesaba sobre las importaciones y la imposición de un recargo mucho menor, que variaba entre 20 y 40 \% según la categoría de vehículo (diferenciados entre vehículos de carga y de pasajeros, y entre estos últimos según la cilindrada). Finalmente, establecía topes máximos de elementos importados determinados por el valor cif de los vehículos.

\section{La industria automotriz bajo el desarrollismo}

Con el arribo de Arturo Frondizi a la presidencia de la República Argentina, el modelo sustitutivo retomó la planificación y la articulación entre la política, la economía y la tecnología. A través de la Ley de Inversiones Extranjeras, los capitales ingresaban al país y luego se redireccionaban a partir de los distintos regímenes especiales y sectoriales. En ese escenario, el sector metalmecánico y en especial la rama automotriz, atrajo una masa de capitales, y maquinaria y equipos, significativa a nivel nacional e incluso regional. A partir de 1960 la radicación de la empresa transnacional comenzó a hacerse efectiva en términos operativos y las firmas comenzaron a sustituir al Estado como motor del crecimiento tecno-productivo. De acuerdo con Chudnovsky y López (1995), desde fines de la década de 1950, las empresas transnacionales lideraron el proceso de sustitución de importaciones en bienes intermedios y de consumo durable, ubicadas estratégicamente en las ramas más dinámicas de la estructura económica.

Hacia mediados de la década de 1960, los resultados del régimen de promoción industrial de 1958 ponían en evidencia el impacto del sector en la economía argentina. El censo industrial de 1964 permite observar que el complejo automotor ocupaba 140000 personas de forma directa, incluyendo la industria terminal, la producción de autopartes, neumáticos, motores de combustión interna, tractores, carrocerías, y talleres de reparación de automóviles y de rectificación de motores. En términos de valor agregado alcanzaba $12 \%$, y teniendo en cuenta el total de las empresas automotrices dos tercios de los empleos eran generados por establecimientos de firmas extranjeras. Estas habían producido un profundo impacto en la estructura económica y social del país, en tanto que, de las diez empresas con mayor facturación en 1964, cinco eran ya automotrices (Sourrouille, 1980). 
Entre 1959 y 1960 el gobierno aprobó la radicación de capitales y los planes de producción de 23 empresas automotrices que se comprometían a producir, entre 1960 y 1961, cerca de 165000 vehículos (Consejo Nacional de Desarrollo, 1966). En pocos años, de las primeras veintitrés firmas registradas (entre las que había algunas muy pequeñas que proponían una escala de producción menor a 100 unidades anuales) sólo operaba la mitad. Diferentes factores propios de la producción automotriz como la baja eficiencia en escalas cortas, la inexperiencia y las dificultades tecnoproductivas, así como los requerimientos de capital y conocimientos intensivos y específicos en el diseño y producción de vehículos, provocaron el cierre de las firmas, cerrando la posibilidad a empresas locales, más asociadas al modelo de desarrollo endógeno de capacidades (Picabea, 2012a). En 1964 dejaron de fabricar automotores las firmas: Autoar, Dinborg, Metalmecánica, Alcre, Cisitalia, Goliath-Hansa, Fábrica de Automóviles Utilitarios, O. Marimón, Panambí, Los Cedros e ITA (véase cuadro 3).

Hacia 1965, la producción automotriz local presentaba resultados acordes a los objetivos de la promoción industrial. Sin embargo, las terminales de capitales locales, en general emprendimientos de baja dotación de capital, no consiguieron sostener la producción y abandonaron la actividad. No es este el lugar para analizar los motivos específicos que en cada caso llevó al cierre de las empresas, pero el rasgo común era su baja escala y escasa experiencia relativa, pero por, sobre todo, el origen de los capitales, en todos los casos, locales.

La producción masiva cubrió los requerimientos del mercado, pero se alejó de las dinámicas socio-técnicas de desarrollo endógeno de capacidades. Especialmente por las restricciones que las terminales ponían a los proveedores locales para la fabricación de autopartes complejas, que las empresas transnacionales terminaban adquiriendo a los proveedores de las casas matrices en sus países de origen, ya sea vía importación o vía radicación local de autopartistas extranjeros. De esta forma, la mayor parte de los aprendizajes tecno-productivos como consecuencia de la interacción entre la industria terminal y autopartista se perdían.

La industria automotriz local se consolida, al menos desde los requerimientos del mercado doméstico e incluso (aunque bajas), en la década de 1970, algunas exportaciones a países limítrofes (véase cuadro 4) (Sourrouille, 1980). A partir de la radicación de las empresas transnacionales, la producción de automóviles aumentó de 40144 unidades en 1959 a 133734 en 1965, más de $200 \%$. En el segmento utilitarios el incremento fue menor (50\%), al igual que en el segmento camiones y colectivos (8\%), pero ello se debió principalmente a una renovación del parque automotor del transporte en los primeros años, que luego se estabilizó.

En 1966, el modelo de industrialización por sustitución de importaciones se encontraba consolidado. Aún frente a los problemas de la balanza de pagos generado por el incremento de la importación de algunos insumos, desde 1964 los ciclos económicos característicos del periodo ya no implicaron caídas absolutas en el producto bruto interno (Basualdo, 2006). El sector automotriz se convirtió en uno de los pilares centrales del modelo de acumulación y a mediados de la década de 1960 representaba $10 \%$ del PBI y $5 \%$ de la fuerza laboral industrial.

El modelo sustitutivo a través de la radicación de empresas transnacionales promovió el desarrollo de un sector manufacturero de bienes durables integrado localmente. Pero la apuesta a la maduración rápida a través de los grandes capitales de empresas extranjeras, configuró una economía altamente concentrada, en cual la oligarquía diversificada y el capital transnacional dominaron estratégicamente los sectores más dinámicos, frente a una burguesía nacional dedicada a las industrias subsidiarias o vegetativas (Basualdo, 2006). 
CUADRO 3. EMPRESAS, UNIDADES PRODUCIDAS

Y RADICACIONES DE CAPITAL ENTRE 1958-1964

\begin{tabular}{lccc}
\hline Empresas & $\begin{array}{c}\text { Años } \\
\text { de operatividad }\end{array}$ & $\begin{array}{c}\text { Unidades } \\
\text { producidas }\end{array}$ & $\begin{array}{c}\text { Radicación de capitales } \\
\text { (en millones de dólares) }\end{array}$ \\
\hline IKA-Renault & 9 & 217000 & 16.5 \\
Fiat & 5 & 75000 & 12 \\
Ford & 5 & 72000 & 34.6 \\
General Motors & 5 & 65000 & 20 \\
Chrysler & 5 & 50000 & 21 \\
Siam Di Tella & 5 & 45500 & 1.4 \\
SAFRAR-IAFA (Peugeot) & 5 & 27000 & 20.5 \\
DINFIA & 12 & 25000 & s. d. \\
Citröen & 5 & 20500 & 8 \\
IASF (Auto Unión) & 5 & 17500 & 1 \\
Mercedes Benz (camiones y ómnibus) & 10 & 13300 & 6.6 \\
Isaard (Gogomobil) & 6 & 15500 & s. d. \\
Metalmecánica & s. d. & 11288 & s. d. \\
Autoar & 10 & 6011 & s. d. \\
Los Cedros & s. d. & 5413 & s. d. \\
Dinborg & 4 & 2729 & s. d. \\
Goliath-Hansa & s. d. & 1570 & s. d. \\
Cisitalia & s. d. & 585 & s. d. \\
Fábrica Automóviles Uti. & s. d. & 528 & s. d. \\
Panambí & s. d. & 370 & s. d. \\
O. Marimón & s. d. & 105 & s. d. \\
ITA & s. d. & 45 & s. d. \\
Alcre & s. d. & s. d. & s. d. \\
\hline
\end{tabular}

Nota: en cursivas las firmas activas hacia 1965.

Fuentes: elaboración propia con base en Adefa (1966) y Sourrouille (1980).

Por otro lado, la creación de un complejo automotor basado en empresas transnacionales generó numerosos impactos negativos en el modelo de acumulación. La radicación de empresas transnacionales modificó cuantitativa y cualitativamente los parámetros de la producción automotriz local, lo que condujo a un cambio en la importancia relativa de la producción estatal de artefactos. Es así que, a comienzos de 1960, Dinfia, la empresa pública pionera del sector, producía 7000 unidades anuales entre utilitarios, automóviles y tractores, mientras que, en el mismo periodo, IKA ya producía 40000 unidades anuales entre automóviles y utilitarios (Picabea, 2012b). Cuando comenzó la producción de las empresas transnacionales, en 1961-1962, la participación de DINFIA pasó a representar menos de $5 \%$ de la producción total de automóviles en Argentina, lo que implicó una caída significativa de la centralidad de la empresa estatal. 


\section{GUADRO 4. PRODUCGIÓN AUTOMOTRIZ ARGENTINA AL AMPARO DE LA REGULACIÓN DE 1958}

\begin{tabular}{lccccccc}
\hline Producción & 1960 & 1961 & 1962 & 1963 & 1964 & 1965 & Totales \\
\hline Automóviles & 40144 & 78274 & 90648 & 75338 & 114617 & 133734 & 532755 \\
Utilitarios & 33506 & 34708 & 25037 & 21119 & 38624 & 43967 & 196961 \\
Camiones y colectivos & 15538 & 23708 & 14195 & 8442 & 13242 & 16835 & 91960 \\
\hline
\end{tabular}

Nota: producción hasta 1965 año en que se produjo una reregulación para el sector automotriz específicamente a partir del Decreto 3642/65.

Fuentes: Adefa (1966) y Sourrouille (1980).

Si bien DINFIA siguió produciendo vehículos y luego, como Industrias Mecánicas del Estado se posicionó como líder del segmento utilitarios hasta 1980, el estilo tecno-productivo de impulso endógeno en 1960 ya no estaba vigente. Las empresas transnacionales no abrieron nunca divisiones de I+D en Argentina por lo que la mayor parte de los procesos productivos y todos los modelos de vehículos fueron siempre diseñados por las casas matrices.

\section{UN MODELO DE ACUMULACIÓN, DOS ESTILOS DE DESARROLLO TEGNO-PRODUCTIVO}

Los regímenes de fomento industrial analizados abarcaron periodos clave en la historia de la industrialización argentina: el peronismo y el desarrollismo. No existen voces contrarias sobre el protagonismo del sector automotriz en el proceso planificado de industrialización por sustitución de importaciones, que inicia con el peronismo y se consolida durante el desarrollismo. Sin embargo, un aspecto poco destacado en la bibliografía es cierta continuidad en las ideas que orientaron toda la trayectoria socio-técnica del periodo, que aquí llamamos tecno-nacionalismo.

Desde la década de 1920 comenzaron a difundirse ideas que asociaban la autonomía nacional con el desarrollo industrial y tecnológico. En ese momento comienza a ponerse en evidencia la impronta de algunos oficiales del ejército argentino, entre los que se destacan Francisco de Arteaga (ideólogo y director de la primera fábrica militar de aviones de América Latina), a Enrique Mosconi (impulsor de la producción local de petróleo y combustibles), y a Manuel Savio (promotor de la siderurgia y la industria pesada). Estas concepciones fueron desarrolladas principalmente por oficiales del ejército argentino y en la década de 1940 se expandió a civiles. De acuerdo con Lalouf (2005), estos grupos de ideología tecno-nacionalista percibían la sustitución de importaciones como un objetivo vinculado a la pretensión de autodeterminación política de la nación. El tecno-nacionalismo orientó un proceso político, económico y tecnológico de coconstrucción de productos y procesos productivos, articuló y creó organizaciones e instituciones que generaron capacidades.

El tecno-nacionalismo orientó la transformación de la estructura económica y comenzó a configurar un conjunto de racionalidades y estrategias que se puso de manifiesto desde 1927 con la creación de una fábrica militar de aviones en Córdoba, confirmando un posicionamiento en el Estado de actores que asociaban el desarrollo industrial y tecnológico con la autonomía nacional. Este pensamiento promovió un doble proceso por el cual la seguridad nacional reforzaba y contribuía con la autodeterminación política, mientras que el desarrollo de la estructura industrial posibili- 
taba la autonomía económica. Con diferencias en los dos periodos considerados en este artículo (el impulso endógeno o exógeno), el proceso de sustitución de importaciones quedó asociado a la idea de autonomía nacional y la función del Estado como conductor y coordinador.

Entre 1944 y 1955, la industrialización quedó definida por la capacidad del Estado de planificar, controlar y participar de forma directa en la actividad económica, lo que generó una transformación significativa en la estructura tecno-productiva y, especialmente, creó las condiciones para la fabricación local de automotores. El Estado generó un conjunto de estrategias tendentes a la endogeneización de capacidades tecno-productivas: infraestructura y fondos públicos, financiamiento al sector privado, formación de recursos humanos calificados, impulso a PyMEs locales, colaboración y radicación de firmas extranjeras especializadas, etc. Esto se observa en los propósitos del régimen de fomento a partir de las industrias de interés nacional desde 1944, en el plan quinquenal de 1947 y en el resto de la regulación sectorial durante todo el gobierno hasta 1955.

La principal característica del primero de los regímenes de promoción fue el impulso endógeno de capacidades. Esto fue aún más explícito a partir de la legislación de 1953. La apertura a inversiones extranjeras de la Ley 14.222/53 no fue, en términos estrictos, muy radical, puesto que implicaba el riguroso control del Estado del proceso de radicación. A partir de una nueva regulación, el gobierno desarrolló un modelo industrial que favorecía el establecimiento de firmas extranjeras, pero asociadas a capitales locales, fueran estos privados o públicos (Picabea y Thomas, 2015b) 9

El estilo socio-técnico de desarrollo endógeno del peronismo permitía que el gobierno tuviera un lugar central en la toma de decisiones, ya que al ocupar un lugar estratégico en las nuevas empresas mixtas podía controlar que el proceso de desarrollo industrial siguiera los objetivos de la política pública. El Estado tuvo un desempeño central a partir de su intervención en el sector industrial, que quedó de manifiesto en los alcances de IAME en la industria automotriz. Los cambios en el modo de regulación permitieron que aquella tuviera un papel preponderante en el modelo de acumulación de industrialización por sustitución de importaciones.

Antes de 1955, las firmas extranjeras Mercedes Benz, Fiat, Kaiser Corp. y Borgward ya producían vehículos en Argentina a partir de acuerdos específicos de radicación, que implicaban la necesaria asociación con el Estado o privados locales. De esta forma, el modelo de industrialización de impulso endógeno permitía al Estado un control del proceso de toma de decisiones, que mantenía la dinámica tecno-productiva dentro de los objetivos propuestos. A comienzos de la década de 1960, sobre la estructura creada por IAME, la industria automotriz creció significativamente (a partir de la radicación de empresas extranjeras), y se convirtió en uno de los ejes del modelo de sustitución de importaciones.

A partir de 1958, con el desarrollismo, se mantuvieron elementos relevantes del pensamiento tecno-nacionalista como la necesidad del desarrollo económico y social, la autonomía económica y la soberanía política. Desde esa perspectiva, el estilo socio-técnico del desarrollismo puede entenderse como una continuidad del tecno-nacionalismo.

\footnotetext{
9 “En la práctica, las empresas mixtas se organizaron bajo diferentes estructuras. Una de ellas surgió de la vinculación de Henry Kaiser con funcionarios de IAME para trasladar la firma - en crisis en Estados Unidos- a Argentina, que concluyó con la creación de Industrias Kaiser Argentina (IKA). Otro modelo fue el que derivó de las relaciones entabladas con Fiat para el asesoramiento en la construcción y la dirección de la Fábrica de Tractores de IAME, que terminó en la creación de la empresa Fiat-Concord en 1954" (Picabea y Thomas, 2015b, p. 72).
} 
Si para el peronismo la independencia política era un objetivo central, expresado en la gestión y el control público del proceso, para el desarrollismo, la estrategia fue la modernización acelerada como motor del incremento de las capacidades tecno-productivas locales. En general, las ideas de Frondizi y Frigerio señalaban, en concordancia con el peronismo, la importancia de generar una estructura industrial capaz de autoabastecer al país de bienes durables intermedios y finales como vía ineludible hacia el desarrollo económico y social. Pero el incremento de las importaciones de determinados insumos industriales, una consecuencia no deseada de la regulación de 1958 y en buena medida contradictoria de la sustitución de importaciones, fue uno de los elementos negativos del régimen. De hecho, algunas empresas locales productoras de bienes de capital no pudieron superar la competencia frente a empresas internacionales, que se beneficiaron a partir del régimen de importación de líneas de producción (Belini y Rougier, 2008).

Otro elemento no contemplado o minimizado del régimen de promoción de 1958 fue la remisión de utilidades de las empresas transnacionales a sus casas matrices. A ello se debió en buena medida, que el flujo de divisas fuera positivo sólo en los primeros años. Las leyes 14780 y 14781 , entre otros aspectos, no contemplaron la generación de patentes locales y, por lo tanto, desestimularon la creación de unidades de I+D locales a diferencia de lo ocurrido con la regulación de 1944 y la creación del Instituto Aerotécnico, primero, y de IAME después (Urcelay, 2015).

Al problema de la falta de consideración de la I+D en el marco regulatorio de 1958, debe sumarse otro efecto no previsto ni detectado a tiempo por la regulación: la sobre y subfacturación de las empresas transnacionales, como medio para remitir más utilidades que las permitidas por el propio régimen de promoción (Schvarzer, 1996).

El efecto negativo sobre las divisas se agravaba por la masa adicional de fondos que las filiales pagaban a sus matrices en concepto de regalías por uso de marcas y tecnologías. De esta forma, el modelo de desarrollo endógeno de capacidades del primer estilo socio-técnico no sólo implicaba ventajas en términos de generación de aprendizajes y capacidades locales; a mediano plazo, también implicaba conservar, fronteras adentro, los costos por pago de patentes.

\section{Conclusiones}

El análisis del marco regulatorio de la industrialización, en general se asocia con resultados macroeconómicos. Esto suele oscurecer procesos que presentan una maduración más lenta, como la generación de capacidades, la formación de recursos humanos o la creación de pequeños establecimientos proveedores que luego articulan toda una trama productiva. El análisis de estas dinámicas socio-técnicas permite repensar algunos supuestos, ya formalizados, sobre la importancia de las políticas públicas o la participación del Estado para alinear y coordinar procesos de desarrollo.

Sobre todo, se pueden cuestionar los abordajes que han relativizado los resultados de la implementación de la política industrial del gobierno peronista respecto de la estructura tecno-productiva y su significación para la posterior radicación de empresas transnacionales durante el desarrollismo. Esto permite complejizar la forma de entender los procesos de cambio tecno-productivos. Por un lado, el artículo señala una clara diferencia en la potencialidad de cada marco regulatorio de acuerdo con su modelo de desarrollo. Por otro lado, la significación que cobra la industria automotriz a partir de 1952 permite establecer continuidades entre el peronismo y el desarrollismo. 
La instalación de las empresas transnacionales favoreció aprendizajes tecnológicos en numerosos aspectos de la estructura industrial argentina (de conocimiento, proceso y producto), sin embargo, a diferencia de los diseños de IAME y otras empresas mixtas, las empresas transnacionales limitaron sus aportes a ciertas capacidades tecno-productivas, pero no generaron aprendizajes en término de I+D.

Por otro lado, la lógica del estilo socio-técnico del desarrollismo favoreció la concentración y centralización de la economía, dejando decisiones estratégicas sobre la matriz insumo-producto sectorial a empresas transnacionales, que buscaban la maximización del lucro y no el desarrollo industrial de una nación. En una estructura económica concentrada y con ciclos, los grandes capitales tenían muchas más oportunidades de crecimiento que las pymes, ya fuera por el apoyo externo de sus filiales o por la adquisición de empresas locales con inconvenientes financieros en las fases de crisis. Esto contribuía a una mayor integración horizontal y vertical, volviendo virtuoso el proceso para los grandes capitales extranjeros.

A diferencia del primer modelo tecno-nacionalista, asociado fuertemente a facciones militares que proponían la generación de aprendizajes, para los desarrollistas, el principal objetivo era la modernización de la economía, por lo que el camino marcado por el intervencionismo estatal directo se entendía como el más largo. Por ello el modelo se caracterizó por la promoción de la inversión extranjera directa y la radicación masiva de empresas transnacionales como aceleradores del proceso. El Estado ya no actuó como interventor directo en la producción, aunque continuó como promotor del desarrollo industrial, a través de diferentes tipos de instrumentos.

A nivel ideológico, el desarrollismo constituyó un proyecto nacionalista y autonomista, pero su dependencia de las inversiones extranjeras disminuyó la capacidad del Estado para controlar el proceso industrializador $\mathrm{y}$, a mediano plazo, las empresas transnacionales torcieron los objetivos originales del tecno-nacionalismo. El resultado fue un estilo socio-técnico que disminuyó significativamente la generación de capacidades y configuró una estructura altamente concentrada, que remitía utilidades a las casas matrices y agravaba aún más los problemas de balanza de pagos del modelo de industrialización sustitutivo.

\section{LISTA DE REFERENCIAS}

Altimir, O., Santamaría, H. y Sourrouille, J. (1967). Los instrumentos de promoción industrial en la postguerra. Desarrollo Económico, 7(26), 149-172. DOI: 10.2307/3465764

Arceo, E. O. (2003). Argentina en la periferia próspera: renta internacional, dominación oligárquica y modo de acumulación. Bernal: Universidad Nacional de Quilmes Ediciones.

Baronson, J. (1971). La industria automotriz en los países en desarrollo: serie de estudios del personal del Banco Mundial. Madrid: Tecnos.

Basualdo, E. M. (2006). Estudios de historia económica argentina: desde mediados del siglo XX a la actualidad. Buenos Aires: Siglo Veintiuno Editores Argentina.

Belini, C. (2006). Negocios, poder y política industrial en los orígenes de la industria automotriz argentina, 1943-1958. Revista de Historia Industrial, 15(31), 109-135. Recuperado de http://revistes.ub.edu/index.php/HistoriaIndustrial/article/view/19663

Belini, C. (2009). La Industria peronista: 1946-1955: políticas públicas y cambio estructural. Buenos Aires: Edhasa. 
Belini, C. (2010). La promoción industrial durante el peronismo. Impacto y límites de la ley de industrias de interés nacional (1944-1958). Temas de historia argentina y americana, 16, 59-97. Recuperado de http://bibliotecadigital.uca.edu.ar/repositorio/revistas/promocion-industrialperonismo.pdf

Belini, C. (2014). Convenciendo al capital. Peronismo, burocracia, empresarios y política industrial, 19431955. Buenos Aires: Imago Mundi.

Biggart, N. W. y Guillen, M. F. (1999). Developing difference: social organization and the rise of the auto industries of South Korea, Taiwan, Spain, and Argentina. American Sociological Review, 64(5), 722. DOI: $10.2307 / 2657373$

Bijker, W. E. (1995). Of bicycles, bakelites, and bulbs: toward a theory of sociotechnical change. Cambridge: MIT Press.

Carvalho, L., Ferraz, J. C. y Kupfer, D. (2009). 50 años en 50 El largo y sinuoso camino del desarrollo industrial de Brasil. Boletín Informativo Techint, 330, 45-72. Recuperado de http://boletin techint.com/pages/ArticuloDetalle.aspx

Catalan, J. (2010). Strategic policy revisited: The origins of mass production in the motor industry of Argentina, Korea and Spain, 1945-87. Business History, 52(2), 207-230. DOI: 10.1080/00 076791003611863

Chudnovsky, D. y López, A. (1995). Promoción y fomento de la innovación tecnológica desincorporada en la industria manufacturera. El caso argentino. Buenos Aires: CENIT.

Consejo Nacional de Desarrollo (Argentina) (1966). La industria automotriz: análisis preliminar. Buenos Aires: Sector Industria y Minería.

Di Tella, G. (1973). La estrategia del desarrollo indirecto. Buenos Aires: Editorial Paidós.

Di Tella, G., Zymelman, M. y Petrecolla, A. (1973). Las etapas del desarrollo económico argentino [por] Guido Di Tella y Manuel Zymelman. Buenos Aires: Editorial Paidós.

Díaz, C. F. (1970). Ensayos sobre la historia económica argentina. Buenos Aires: Amorrortu.

Ferrer, A. (2004). La economía argentina: desde sus origenes hasta principios del siglo XXI (3a. ed.). Buenos Aires: Fondo de Cultura Económica.

Gerchunoff, P. y Llach, L. (2003). Ved en torno a la noble igualdad: crecimiento, equidad y política económica en Argentina, 1880-2003 (Fundación para la integración de la Argentina en el mundo No. 2003-003). Buenos Aires: Fundación Proyecto Educación y Nuevas Tecnologías. Recuperado dehttps://crecimientoeconomico-asiain.weebly.com/uploads/1/2/9/0/1290958/llach_y_ger. chunoff_ved_en_trono_a_la_noble_igualdad.pdf

Harari, I. (2007). Crónica de una privatización anunciada: alcances y límites de la producción automotriz bajo el peronismo. Anuario CEICS, 1(1), 47-70.

Harari, I. (2015). A media máquina: procesos de trabajo, lucha de clases y competitividad en la industria automotriz argentina (1952-1976). Buenos Aires: Ediciones RYR.

Ianni, V. L. (2008). La especificidad del desarrollo de la industria automotriz en la Argentina, 1959-1963. Estudos Ibero-Americanos, 34(2). DOI: 10.15448/1980-864X.2008.2.4506

Jorge, E. F. (1971). Industria y concentración económica: desde principios de siglo hasta el peronismo. Siglo XXI. Buenos Aires.

Katz, J. y Kosacoff, B. (1989). El proceso de industrialización en la Argentina: evolución, retroceso y prospectiva. Buenos Aires: Centro Editor de América Latina/CEPAL.

Lalouf, J. (2005). Construcción y desconstrucción de un caza nacional. Análisis socio-técnico de la experiencia de diseño y producción de los aviones Pulqui I y II (Argentina-1946/1960) (Tesis de maestría). Universidad Nacional de Quilmes, Argentina. 
Llach, J. J. (1984). El plan pinedo de 1940, su significado histórico y los orígenes de la economía política del peronismo. Desarrollo Económico, 23(92), 515. DOI: 10.2307/3466422

Mallon, R. D. y Sourrouille J. V. (1973). La política económica en una sociedad conflictiva: el caso argentino. Buenos Aires: Amorrortu. Recuperado de http://biblio.econ.uba.ar/cgi-bin/koha/opacdetail.pl?biblionumber $=117148$

Picabea, F. (2012a). Apogeo, inercia y caída del proyecto metalmecánico tecno-nacionalista: el caso de Industrias Mecánicas del Estado (Argentina 1952-1980) (PhD. Tesis). Universidad de Buenos Aires, Buenos Aires.

Picabea, F. (2012b). Cambios en la estrategia de desarrollo tecno-productivo en Argentina: trayectoria de una empresa pública en el auge de las transnacionales: DINFIA (1955-1967). En ESOCITE IX Jornadas Latinoamericanas de Estudios Sociales de la Ciencia y la Tecnología (pp. 288329). Ciudad de México: s. e.

Picabea, F. y Thomas, H. (2015a). Autonomía tecnológica y desarrollo nacional: historia del diseño y producción del Rastrojero y la moto Puma. Buenos Aires: Editorial Atuel.

Picabea, F. y Thomas, H. (2015b). Tecnología y política: historia del rastrojero y la moto puma. Buenos Aires: Universidad Nacional de Quilmes.

Raccanello, M. (2013). Industrias aeronáuticas y mecánicas del estado y la lógica de la política económica peronista. América Latina en la Historia Económica, 20(2), 177. DOI: 10.18232/alhe.v20i2.543

Schvarzer, J. (1987). Promoción industrial en Argentina: características, evolución y resultados. Buenos Aires : CISEA. Recuperado de http://biblio.econ.uba.ar/cgi-bin/koha/opac-detail.pl?biblion umber $=123267$

Schvarzer, J. (1996). La industria que supimos conseguir. Buenos Aires: Planeta.

Sourrouille, J. V. (1980). Transnacionales en América Latina el complejo automotor en Argentina. México: Nueva Imagen. Recuperado de http://biblio.econ.uba.ar/cgi-bin/koha/opac-detail.pl?biblio number $=123168$

Thomas, H. (1999). Dinâmicas de inovação na Argentina (1970-1995): abertura comercial, crise sistêmica e rearticulação (Tesis de doctorado). Universidade Estadual de Campinas, Brasil.

Thomas, H. (2006). Trayectorias socio-técnicas y estilos de cambio tecnológico en países subdesarrollados: la resignificación de tecnologías (América Latina, 1930-2006). En XX Jornadas Argentinas de Historia Económica. Mar del Plata: Asociación Argentina de Historia Económica/Universidad de Quilmes.

Thomas, H. (2008). Estructuras cerradas versus procesos dinámicos: trayectorias y estilos de innovación y cambio tecnológico. En H. Thomas y A. Buch (eds.), Actos, actores y artefactos. Sociología de la tecnología (pp. 217-262). Bernal: Universidad Nacional de Quilmes.

Urcelay, F. (2015). El pensamiento tecno-nacionalista en el inicio de la política de desarrollo industrial. La creación del Instituto Aerotécnico en 1943. En VI Jornadas de la División Historia. Luján: Universidad Nacional de Luján.

\section{Documentos}

Asociación de Fabricantes de Automotores (Adefa), Anuarios, 1966.

DPEN 14.630 de 1944, INFOLEG, Ministerio de Economía de la Nación (MEN).

DPEN 25.056 de 1951, INFOLEG, (MEN).

DPEN 6.191 de 1952, INFOLEG, (MEN).

DPEN 3.693 de 1959, INFOLEG, (MEN). 
IKA, Memoria y balance general, 1960 .

Ley Nacional 14.222 de 1953, INFOLEG, MEN.

Ley Nacional 14.780 de 1958, INFOLEG, MEN.

Ley Nacional 14.781 de 1958, INFOLEG, MEN. 